repeated, examining education instead of social class. Approval was obtained from the ethics committee, and participants gave written informed consent.

Being in the manual social class $(26 \%)$ was associated with a significantly higher prevalence of calcification (odds ratio $=2.3,95 \%$ confidence interval 1.3 to $5.2, \mathrm{P}=0.04$ ), as was having left full time education before the age of 19 (odds ratio 2.8 (1.2 to 6.3), $\mathrm{P}=0.01$ ). Adjusting for age, sex, systolic blood pressure, high density lipoprotein cholesterol, low density lipoprotein cholesterol, triglycerides, alcohol consumption, and body mass index either singly or simultaneously attenuated the odds ratios for social class (adjusted odds ratio $=2.0,95 \%$ confidence interval $(0.7$ to 5.2 ), $\mathrm{P}=0.2$ ) and educational status (adjusted odds ratio $2.2(0.8$ to 6.0$), \mathrm{P}=0.1)$ only slightly, although their significance was reduced. Adjusting for pack years of smoking and physical activity level in those 126 participants on whom these data were available did not alter the odds ratio. In this subgroup the odds ratio for social class was 1.8 and was 3.0 for educational status, both before and after adjustment.

\section{Comment}

The study shows that socioeconomic differences in coronary artery calcification already exist in men and women in their $30 \mathrm{~s}$. A socioeconomic difference in the precursor non-calcified lesions of atherosclerosis may be present even earlier in the life course. Social class differences in coronary risk factors were generally small or non-existent in this cohort (data not given) and explained little of the social class difference in coronary artery calcification.

The unequivocal class difference in people in their 30s has important implications. Firstly, interventions aimed at reducing inequalities in heart disease must include young adults and possibly children. Secondly, studies of socioeconomic gradients in coronary heart disease that do not consider the risk factor profiles of participants in their 20s and 30 s are unlikely to explain the gradient in full. Thirdly, the paucity of effect of adjusting for established risk factors on class difference emphasises that the biological mechanisms through which social inequalities affect risk for coronary heart disease have yet to be discovered. Finally, electron beam computerised tomography is an important technique for exploring the basis of socioeconomic differences in coronary disease in relatively young cohorts.

Contributors: HMC initiated the research, designed the protocol, participated in the data collection, analysed the data, and drafted the paper. She is also the guarantor. MBR helped formulate the hypothesis and study design, advised on the protocol, participated in the data collection, edited the paper, and scored all the electron beam computerised tomography scans. SRU and JHF helped formulate the hypothesis and study design, advised on the protocol, and edited the paper.

Funding: Project grant from the British Heart Foundation.

Competing interests: None declared.

1 Brunner E, Shipley MJ, Blane D, Smith GD, Marmot MG. When does cardiovascular risk start? Past and present socioeconomic circumstance and risk factors in adulthood. I Epidemiol Community Health 1999;53: $757-64$.

2 Frankel S, Smith GD, Gunnell D. Childhood socioeconomic position and adult cardiovascular mortality: the Boyd Orr cohort. Am J Epidemiol 1999;150:1081-4.

3 Leeson CP, Whincup PH, Cook DG, Mullen MJ, Donald AE, Seymou CA, et al. Cholesterol and arterial distensibility in the first decade of life a population-based study. Circulation 2000;101:1533-8.

4 Colhoun HM, Rubens MB, Underwood SR, Fuller JH. The effect of type 1 diabetes mellitus on the sex difference in coronary artery calcification. $J$ Am Coll Cardiol (in press).

5 Wexler L, Brundage B, Crouse J, Detrano R, Fuster V, Maddahi J, et al. Coronary artery calcification: pathophysiology, epidemiology, imaging methods, and clinical implications. A statement for health professionals from the American Heart Association. Circulation 1996;94:1175-92.

(Accepted 11 August 2000)
Royal Brompton and Harefield NHS Hospital Trust, London SW3 6NP Michael B Rubens consultant radiologist S Richard Underwood professor of cardiac imaging

Correspondence to: $\mathrm{H}$ M Colhoun helen@public-

health.ucl.ac.uk

\title{
Corneal donation in the accident and emergency department: observational study
}

\author{
J Long, D Walsh, D A W Ritchie, F Russell
}

Corneal grafting restores sight to individuals with corneal damage. Corneal donations have decreased recently from 4419 in 1996 to 3346 in $1998 .{ }^{1}$ Patients pronounced dead in accident and emergency departments are potential donors of corneas for 24 hours, but this resource is underused. ${ }^{2}$ In the year before the study only one pair of corneas ( 1 of 106 deaths $(0.9 \%)$ ) was donated in our department.

The study consisted of developing a policy to request consent for corneal donation and to determine whether the rate of corneal donation was affected. In addition, a simple questionnaire assessed relatives' attitudes to corneal donation.

\section{Methods and results}

All patients pronounced dead in the accident and emergency department from April to July 1999 were considered for the study. Exclusion criteria were suspi- cious deaths, patients $<16$ years old, contraindication to corneal donation (scarring or deterioration of tissue, infectious disease in the eye tissue, rare invasive brain tumour, Alzheimer's disease or other disease of unknown aetiology), or no relatives present within six hours of death.

All relatives were asked whether the patient carried a donor card or had expressed a wish to donate organs, including corneas. Once consent was granted, we then checked whether there were any contraindications. The transplant coordinator was contacted and the relatives of patients in the study were asked two simple questions in a questionnaire: whether they thought it was appropriate to be approached about corneal donation in accident and emergency; and whether they were offended or distressed by the request.

During the four month study period 47 deaths occurred in the department. Eleven patients were excluded, comprising six suspicious deaths, three with 
Royal Alexandra Hospital, Paisley PA2 9PN

F Russell

consultant in accident and emergency

Correspondence to: J Long jason.long@ ntlworld.com no next of kin, and two with known contraindications. In addition, 11 patients were missed by the study. Of the remaining 25 patients, consent was given for nine and nine pairs of corneas were retrieved for storage.

One questionnaire was incorrectly completed, leaving 24 for analysis. Twenty one relatives thought it was appropriate to be approached regarding corneal donation; 23 were not distressed by the request.

Only four out of 24 patients carried a donor card or had expressed a wish to their relatives to donate their organs and in each case this included corneas. These data may add to the political debate on the validity of donor cards and the need for an opt out policy. Of the nine patients who donated corneas, three carried a donor card.

\section{Comment}

The study shows that an active policy for corneal donation in accident and emergency departments can have a dramatic effect on retrieval rates of corneas. The retrieval rate of $36 \%$ (25\% if the 11 missed patients are included) compares favourably with rates found in previous studies. ${ }^{3-5}$ The rate is also a significant increase from the $1 \%$ of the previous year $(95 \%$ confidence difference $24 \%(10 \%$ to $38 \%) ; \mathrm{P}=0.001)$.

Many factors may be responsible for the 11 patients missed by the study. Despite training, staff may have felt it was inappropriate to approach relatives at this time of grief, or the department may have been busy, causing concern that the procedure would be time consuming. Exclusion criteria may have been known but not documented in the case notes at the time of death.
More work is needed to educate the public and staff about corneal donation. In January 1999 the Manchester Eye Bank was unable to issue any corneas for grafting because of a shortage. In 1998, 28 pairs of corneas were retrieved in the west of Scotland. Projecting our results for one year, we would expect to double the number of corneas donated in the west of Scotland simply by implementing this policy in one accident and emergency department. Accident and emergency is clearly a specialty that could help resolve the national shortage of donor corneal tissue.

We thank all the medical and nursing staff in the Accident and Emergency Department, Victoria Infirmary, Glasgow, for their help and support during this study.

Contributors: JL had the original idea for the study, coordinating it, participating in data collection and interpretation, and writing the paper. He is also the guarantor. DW participated in the design of the study and data collection. DAWR participated in the design of the study. FR participated in the design of the study, data collection and interpretation, and writing the paper. Funding: None.

Competing interests: None declared.

1 United Kingdom Transplant Support Service Authority. Statistics prepared by UK Transplant Support Service Authority from the National Transplant Database maintained on behalf of the UK transplant community. Bristol: UKTSSA, 1999.

2 Magrath HP, Boulstridge LJ. Tissue donation after death in the accident and emergency department: an opportunity wasted? J Accid Emerg Med 1999;16:117-9.

3 Lowenstein A, Rahmeil R, Varsanno D, Moshe L. Obtaining consent for eye donation. Israel J Med Sci 1991;27:79-81.

4 Diamond GA, Campion M, Mussoline JF, D'Amico RA. Obtaining consent for eye donation. Am J Ophthalmol 1987;302:149-53.

5 Lewis LM, Martin L, Hoffman T, Ruoff BE. Tissue and organ procurement in the emergency department setting. Am J Emerg Med 1993;11:347-9.

(Accepted 22 May 2000)

\section{A memorable patient \\ Not an ordinary sore throat}

It was a busy Saturday morning surgery. The patient was a 52 year old Menorcan waiter whom I knew vaguely, having seen him a few times when his marriage was in trouble. His new partner made the call at about 1030 am when there were still 15 patients to see and three visits in the book. He had been down to the surgery the previous afternoon with a sore throat without a cough, and had been prescribed penicillin by one of my partners. His girlfriend said that he was no better and was having trouble breathing. I asked if he could come down to the surgery, to which the reply was: "No, he finds it very difficult to get down the stairs." I thought it best to speak directly to the patient.

He apparently had a slight sore throat, but was not wheezy, and could talk normally with no obvious breathing problem. There was no history of asthma or allergy, and he said that he felt fine in bed but could really not get down the stairs; he even found it an effort to get to the lavatory. Somewhat reluctantly I said that I would visit after surgery.

He looked fine lying in bed, and conversed easily with no dyspnoea or stridor. "I'm sorry to call you out, Doctor, but I cannot get down the stairs."

Impatiently, I asked him to sit up so that I could look in his throat and examine his chest.

"I can't, Doctor."

"Of course you can," I said, firmly helping him upright, at which moment he choked and gasped, and threw himself back flat on the bed. I asked him to try again and the same thing happened. He seemed to be unable to breathe in the vertical position but was fine lying flat.
I examined him flat on the bed: the pharynx was a little red, but there was no quinsy or obvious swelling, and no cervical lymphadenopathy. He had a slight temperature. The lungs were clear and there was no asymmetry in expansion. He could breathe equally well lying on the right or the left, or on his back.

Finally, taking him seriously, I asked if he had any problems in the past with his throat, and the reply was that when he was a child he had had some throat polyps removed.

I gave him some steroids and arranged to admit him, with a provisional anatomical diagnosis, and with strict instructions to the ambulance crew to keep him flat when going down the stairs. The ear, nose, and throat registrar had said: "Send him in-it sounds absolutely fascinating."

Initial laryngoscopy proved difficult, but after 48 hours of steroids and antibiotics he had his posterior laryngeal polyp excised-arising from the arytenoids. Results of histology were benign.

The usual response to laryngeal obstruction-usually caused by epiglottic or pharyngeal swelling-is to sit up and forward. This man had significant laryngeal obstruction from a posterior polyp, which flopped in and out of the glottic opening as he changed position from horizontal to vertical and back again.

The image of my patient's reaction to "iatrogenic" laryngeal obstruction when I forced him into the vertical position to examine him will certainly live with me for a long time.

Jim Price general practitioner, Chichester 